\title{
Flat-Gain Fiber Raman Amplifiers Using Equally Spaced Pumps
}

\author{
Jonathan Hu, Student Member, IEEE, Student Member, OSA, Brian S. Marks, Member, IEEE, Member, OSA, and \\ Curtis R. Menyuk, Fellow, IEEE, Fellow, OSA
}

\begin{abstract}
This paper analyzes the gain flatness of multiwavelength pumped fiber Raman amplifiers using equally spaced pumps with both a fixed and an optimized central pump wavelength. The signal gain ripple using equally spaced pumps is compared with the case in which the pump wavelengths are allowed to vary for two, four, and eight pumps with 20-, 40-, and 80-nm signal bandwidths. The paper shows that using an optimized central pump wavelength with equal pump spacing simplifies system design, while the gain ripple is no more than $0.4 \mathrm{~dB}$ larger than the ripple obtained when the pump wavelengths are optimized for the cases considered.
\end{abstract}

Index Terms-Modeling, optical fiber amplifiers, optical fiber communication, Raman scattering.

\section{INTRODUCTION}

$\mathbf{T}$ HE capacity of wavelength-division-multiplexed (WDM) systems is limited by the gain bandwidth of erbium-doped fiber amplifiers (EDFAs). Multiple-pump fiber Raman amplifiers (FRAs) can increase the transmission bandwidth. Moreover, Raman amplifiers have better noise performance than EDFAs because the amplification is distributed. While multiple pumps can be used to equalize gain over a large bandwidth, it has been a challenge to design FRAs with flat gain [1]. Recently, a novel optimization procedure has been developed that allows for a choice of pump frequencies and powers in order to achieve the best possible gain flatness over a specified signal bandwidth [2], [3]. This optimization procedure chooses not only optimal pump powers, but also their wavelengths, allowing any possible values within a realistic range of values. While this procedure corresponds to the best one can do to flatten the gain using a fixed number of pumps, we have found that the procedure often chooses an optimum in which two pump wavelengths are very close to one another. However, using cascaded couplers to combine two closely spaced wavelengths may be an engineering challenge, as has been pointed out by other authors [4]. In addition, spacing the pump wavelengths evenly yields a simpler design for the Raman amplifier and may be desirable. Hence, it is often

Manuscript received September 16, 2003; revised March 15, 2004. This work was supported in part by the U.S. Department of Energy (DOE) and the Nationa Science Foundation (NSF).

J. Hu and C. R. Menyuk are with the Department of Computer Science and Electrical Engineering, University of Maryland Baltimore County, Baltimore MD 21250 USA (e-mail: hu1@umbc.edu).

B. S. Marks is with with the Department of Computer Science and Electrical Engineering, University of Maryland Baltimore County, Baltimore, MD 21250 USA and also with the Laboratory for Physical Sciences, College Park, MD 20740 USA (e-mail: marks@umbc.edu).

Digital Object Identifier 10.1109/JLT.2004.829187 used in practice. In fact, a recent experiment has demonstrated $80 \mathrm{~nm}$ of flat gain using four equally spaced Raman pumps [5]. Another potential drawback of the method outlined in [2] and [3] is that the optimal wavelengths chosen by the algorithm may not be commercially available. In this case, a system designer would like to determine the best possible gain ripple obtainable by fixing the pump wavelengths to a set of specified values that are available from manufacturers.

This paper extends the optimization procedure of Perlin and Winful [2], [3] to the case of a priori specified pump wavelengths and specifically considers the impact of spacing the pumps evenly in wavelength. The procedure in this paper differs from that of [2] in that the pump wavelengths are constrained to chosen values and only their corresponding powers are optimized. In addition, an iteration procedure is used that relies on a shooting algorithm to optimize the input pump powers [6]. It is found that a Raman amplifier with equally spaced pumps exhibits gain flatness on the same order as one with optimized pump wavelengths. Allowing both the central wavelength and the wavelength spacing of the pumps to be optimized yields a gain ripple that is within $0.4 \mathrm{~dB}$ for all the cases that were considered when compared with the completely wavelength optimized case, as in [2] and [3].

\section{DESIGN AlgORITHM}

First, we describe wave propagation in the backward-pumped multiple-pump FRA using a system of coupled equations that neglect the effects of spontaneous Raman scattering and Rayleigh backscattering, since these noise effects do not affect the amplifier gain profile [7]. We only consider the steady-state gain, as is appropriate when modeling long-haul systems. In networks that add and drop channels, transient gain would have to be considered as well [8], [9]. The pump-to-pump, pump-to-signal, and signal-to-signal Raman interactions are considered in the coupled equations

$$
\pm \frac{d P_{k}}{d z}=-\alpha_{k} P_{k}+\sum_{j=1}^{n+m} g_{j k} P_{k} P_{j}
$$

where $n$ is the number of pump waves, and $m$ is the number of signal waves. The values $P_{k}, \nu_{k}$, and $\alpha_{k}$ describe, respectively, the power, frequency, and attenuation coefficient for the $k$ th wave, where $k=1,2, \ldots, n+m$. The gain coefficient $g_{j k}$ describes the power transferred by stimulated Raman scattering between the $j$ th and $k$ th waves and is given by $g_{j k}=\left(1 / 2 A_{\text {eff }}\right) g_{j}\left(\nu_{j}-\nu_{k}\right)$ for $\nu_{j}>\nu_{k}$ and $g_{j k}=-\left(1 / 2 A_{\mathrm{eff}}\right)\left(\nu_{k} / \nu_{j}\right) g_{k}\left(\nu_{k}-\nu_{j}\right)$ for $\nu_{j}<\nu_{k}$, 

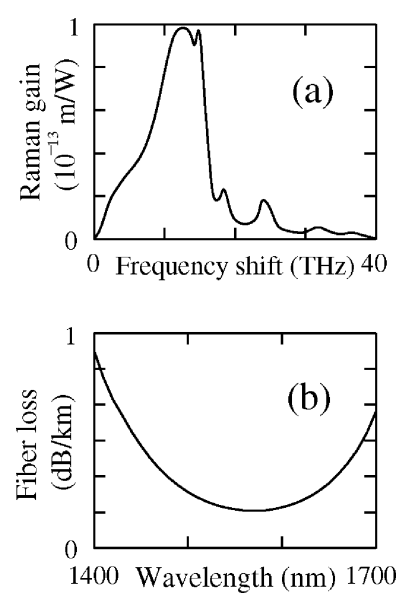

Fig. 1. (a) Raman gain spectrum $g_{R}(\Delta \nu)$ of a typical silica fiber at the pump wavelength $\lambda_{0}=1 \mu \mathrm{m}$. (b) Loss profile for a typical silica fiber. These curves are the profiles used in our simulations to obtain the results in Section III and Fig. 2.

where $g_{i}(\Delta \nu)$ is the Raman gain spectrum measured at the pump frequency $\nu_{i}$, shown in Fig. 1 for a silica fiber at $1 \mu \mathrm{m}$, and $A_{\text {eff }}$ is the fiber effective area. Although we use the particular gain-and-loss profile for $g_{j}(\Delta \nu)$ and $\alpha_{j}$ shown in Fig. 1, the optimization algorithm presented in this paper does not rely on the choice of these profiles. For a fiber span of length $L$, the boundary conditions are defined at $z=L$ for the backward-propagating pump waves $P_{k}(L)=P_{k 0}(k=1,2, \ldots, n)$ and at $z=0$ for the signal waves $P_{k}(0)=P_{k 0}(k=n+1, n+2, \ldots, n+m)$.

The gain for every signal channel $k=n+1, n+2, \ldots, n+m$ can be expressed in terms of the integrals $I_{j}=\int_{0}^{L} P_{j}(z) d z$, $j=1,2, \ldots, n+m$ as [2]

$$
\begin{aligned}
G_{k} & \equiv \frac{P_{k}(L)}{P_{k}(0)} \\
& =\exp \left(-\alpha_{k} L+\sum_{j=n+1}^{n+m} g_{j k} I_{j}\right) \exp \left(\sum_{j=1}^{n} g_{j k} I_{j}\right) \\
& =G_{L, k} G_{G, k} .
\end{aligned}
$$

The first exponential factor in (2) $G_{L, k}$ represents the effect of fiber attenuation and signal-to-signal Raman scattering, whereas the second term $G_{G, k}$ describes the Raman gain due to pump-to-signal Raman scattering experienced by the channel $k$. To flatten the gain at all signal wavelengths, we must have $G_{k} \cong 1$ for all $k$. Assuming that the factors $G_{L, k}$ are known, the approach that we use to find the pump combination corresponding to the flattest net gain breaks into two parts: 1) a genetic algorithm [10] and 2) an iterative integration procedure. The genetic algorithm searches for the integral of the pump power $I_{j}$ that corresponds to the flattest possible gain [2], [3]. Starting with a randomly chosen population of individuals (genesis), each with a set of $\left\{I_{j}\right\}_{j=1}^{n}$, we iteratively apply the three evolution procedures: crossover, mutation, and natural selection. On each iteration step, we select the most fit $20 \%$ of the population and repeat it four times to form the next generation. The size of the elite population (20\%) was chosen

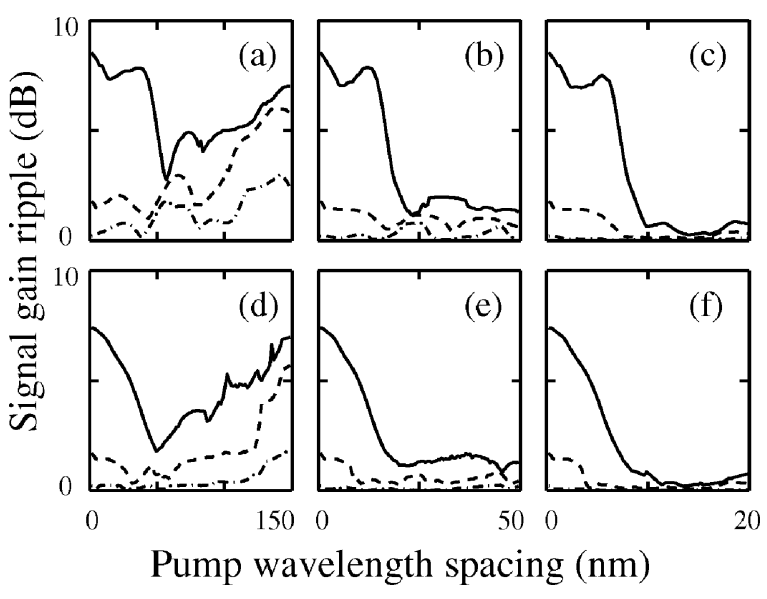

Fig. 2. Signal gain ripple as a function of wavelength spacing using: (a) and (d) two pumps; (b) and (e) four pumps; and (c) and (f) eight pumps. Dotted-dashed, dashed, and solid curves show signal bandwidths of 20, 40, and $80 \mathrm{~nm}$, respectively. (a)-(c) shows gain ripple when the pumps' central wavelength is fixed at $1450 \mathrm{~nm}$, and (d)-(f) shows results when the pumps' central wavelength is optimized.

to be large enough so that the algorithm successfully avoids being trapped in local optima of the sample space but small enough to obtain a result quickly. We then randomly form couples among them and swap a randomly chosen $50 \%$ of the parameters between the members of each couple (crossover). Mutation is implemented by small random changes in one or more parameters of each individual. The iterative procedure continues until the population fitness level does not improve further, and the best individual is chosen as the final set of integrated pump powers $\left\{I_{j}\right\}_{j=1}^{n}$. If we allow the central pump wavelength to be simultaneously optimized, we can simply include it as an additional parameter of each individual. We tried different simulations with redundant iteration and have not observed any cases in which the algorithm was trapped in a local optimum of the search space. At the second stage of the design procedure, the iterative integration procedure then solves (1) simultaneously for all pumps and signals using a multiple shooting algorithm to determine the appropriate input pump powers corresponding to flat gain [6].

In this paper, we use a similar genetic algorithm as in [2] to select the $I_{j}$ values for each pump, but we initially set the number of pumps and their wavelength spacing to values we choose $a$ priori. Therefore, in the case of a fixed central wavelength, the pump wavelengths are chosen and fixed, while in the case of the optimized central wavelength, the wavelength spacing is fixed, but the pump wavelengths are strictly determined by the central wavelength, which is optimized by the genetic algorithm. Although the wavelengths could have any value, we chose them to be evenly spaced for consistency with experimental work to date [4], [5]. Choosing the pumps to be evenly spaced simplifies the design and aids initial coupling into the systems in the experiments [11]. In contrast with the integration procedure used in [2], we used a shooting algorithm [6] to arrive at the input pump powers once the $I_{j}$ values were obtained from the genetic algorithm. We then changed the pumps' wavelength spacing and optimized the pump powers again for each spacing to investigate the effect on the amplifier's gain ripple. 


\section{RESULTS AND DISCUSSION}

In Fig. 1, we plot the Raman gain spectrum of a silica fiber pumped at $1 \mu \mathrm{m}$. Using this gain spectrum, we apply the iterative algorithm described previously to find the signal gain ripple for various values of pump wavelength spacing $\Delta \lambda$, and we plot the results in Fig. 2(a)-(f). In Fig. 2(a)-(c), the central wavelength of the pumps is $1450 \mathrm{~nm}$. We use $1450 \mathrm{~nm}$ because the peak of the Raman gain spectrum in standard fiber is about 100 $\mathrm{nm}$ higher than the pump, and $1550 \mathrm{~nm}$ is in the middle of our signal spectrum and a typical $C$-band value. In Fig. 2(d)-(f), the central wavelength is optimized. The signals were taken to be continuous waves with a wavelength separation of $0.8 \mathrm{~nm}$ and a power of $-3 \mathrm{dBm}$ per wave. We considered three signal bandwidths: 1540-1560 nm corresponding to the dotted-dashed curves; 1530-1570 nm corresponding to the dashed curves; and 1525-1605 nm corresponding to the solid curves. Fig. 2(a) and (d), (b) and (e), and (c) and (f) correspond to using two-, four-, and eight-pump wavelengths, respectively. We simulate propagation through a 50-km span of fiber that is backward-pumped and has a typical attenuation spectrum [12].

In Fig. 2(a)-(c), which uses a fixed central wavelength, we first note that the signal gain ripple is large for the $80-\mathrm{nm}$ signal bandwidth when the pump spacing is small. The large gain ripple is expected since all the pumps are clustered together and are therefore only able to flatten the gain over a small bandwidth in the broad signal spectrum. In Fig. 2(a)-(c), we also note that there are multiple local minima in the gainripple curves, especially in the case of two pumps, shown in Fig. 2(a). This behavior is a consequence of the shape of the Raman gain spectrum shown in Fig. 1, which itself has many local minima.

If we consider the 80-nm signal bandwidth in Fig. 2(a), as the wavelength spacing increases with the central wavelength fixed, the Raman amplifier is able to achieve a relatively flat gain across the entire signal spectrum only at $\Delta \lambda \cong 55 \mathrm{~nm}$. The sharp decrease of the gain ripple just to the left of this first minimum is due to the sharp change of the Raman gain spectrum to the right of the maximum value shown in Fig. 1. When the peak of the Raman gain curve is just less than the edge of the signal bandwidth, there will be large gain ripple at the high-wavelength edge of the signal bandwidth. Increasing the pump wavelength spacing so that the maximum Raman gain corresponds with the edge of the signal spectrum then leads to minimum signal gain ripple. When the wavelength spacing is further increased, it becomes more difficult to flatten the gain because of the sloped shape of the Raman gain spectrum. Therefore, the gain ripple increases.

Fig. 2(d)-(f) shows results of the gain ripple for equally spaced pumps with an optimized central wavelength. Compared with the corresponding plot in Fig. 2(a)-(c) for the $80-\mathrm{nm}$ signal bandwidth curve, the curves of gain ripple drop smoothly at lower wavelength spacing as the pump spacing increases due to the optimization of the central wavelength. Allowing the algorithm to vary the central wavelength gives more flexibility to search for a set of pump wavelengths corresponding to low
TABLE I

Signal GAIN RIPPLE FOR DifFERENT ALGORITHMS EXPRESSED IN DECIBELS. CASES I, II, AND III REPRESENT THE CASES OF EQUally SPACED PuMPS With a Central WaVelength of $1450 \mathrm{~nm}$, EQually SPACED Pumps With aN Optimized Central WaVelength, AND Freely Optimized PuMP WAVELENGTHS, RESPECTIVELY

\begin{tabular}{c|c|c|c|c}
\hline $\begin{array}{c}\text { \# of } \\
\text { pumps }\end{array}$ & $\begin{array}{c}\text { Signal } \\
\text { Bandwidth }\end{array}$ & Case I & Case II & Case III \\
\hline \multirow{3}{*}{2} & $20 \mathrm{~nm}$ & $0.180 \mathrm{~dB}$ & $0.039 \mathrm{~dB}$ & $0.038 \mathrm{~dB}$ \\
\cline { 2 - 5 } & $40 \mathrm{~nm}$ & $1.015 \mathrm{~dB}$ & $0.315 \mathrm{~dB}$ & $0.288 \mathrm{~dB}$ \\
\cline { 2 - 5 } & $80 \mathrm{~nm}$ & $2.770 \mathrm{~dB}$ & $1.786 \mathrm{~dB}$ & $1.706 \mathrm{~dB}$ \\
\hline \multirow{3}{*}{4} & $20 \mathrm{~nm}$ & $0.019 \mathrm{~dB}$ & $0.014 \mathrm{~dB}$ & $0.010 \mathrm{~dB}$ \\
\cline { 2 - 5 } & $40 \mathrm{~nm}$ & $0.435 \mathrm{~dB}$ & $0.272 \mathrm{~dB}$ & $0.072 \mathrm{~dB}$ \\
\cline { 2 - 5 } & $80 \mathrm{~nm}$ & $1.167 \mathrm{~dB}$ & $0.833 \mathrm{~dB}$ & $0.435 \mathrm{~dB}$ \\
\hline \multirow{3}{*}{8} & $20 \mathrm{~nm}$ & $0.008 \mathrm{~dB}$ & $0.006 \mathrm{~dB}$ & $0.004 \mathrm{~dB}$ \\
\cline { 2 - 5 } & $40 \mathrm{~nm}$ & $0.080 \mathrm{~dB}$ & $0.064 \mathrm{~dB}$ & $0.044 \mathrm{~dB}$ \\
\cline { 2 - 5 } & $80 \mathrm{~nm}$ & $0.255 \mathrm{~dB}$ & $0.196 \mathrm{~dB}$ & $0.146 \mathrm{~dB}$ \\
\hline \multirow{2}{*}{ Case I $=$} \\
e equally spaced pumps with a central \\
wavelength of 1450 nm wase II = equally spaced pumps with an \\
optimized central wavelength \\
Case III = freely optimized pump wavelengths
\end{tabular}

gain ripple when the pump spacing is specified. We also note that the local minima in Fig. 2(d)-(f) are not as prominent due to the optimization of the central wavelength.

Note also that when we use eight pumps, we obtain very flat gain when the pump spacing is greater than $10 \mathrm{~nm}$, regardless of the signal bandwidth. This case is therefore very insensitive to wavelength spacing. If one uses two or four pumps, however, it is best to choose the optimal pump wavelength values. The typical input pump powers that the simulation produces are tens to hundreds of milliwatts. For most realistic cases, these values are reasonable.

In Table I, we compare our results for equally spaced pumps with the case in which both pump powers and wavelengths are allowed to be simultaneously optimized with no constraints [2], [3]. The values in the columns of the table corresponding to cases I and II are the minimum signal gain-ripple value obtained in Fig. 2 for equally spaced pumps with fixed and optimized central wavelength, respectively. The final column (case III) shows the signal gain ripple obtained when the pump wavelengths were allowed to be optimized. Note that for two pumps, cases II and III are the same. Table I shows that when the central wavelength is optimized, the constraint of equally spaced pumps yields gain flatness that is nearly the same as when the pump wavelengths are optimized. Using equally spaced pumps simplifies multiple-pump FRA design. For the cases considered in this paper, the maximum difference of gain ripple between cases I and III is approximately $1 \mathrm{~dB}$, while between cases II and III, it is about $0.4 \mathrm{~dB}$.

\section{CONCLUSION}

This paper demonstrated a method to design flat-gain multiwavelength-pumped FRAs using equally spaced pumps with either a fixed or optimized central pump wavelength. The paper made this comparison for two, four, and eight pumps 
with 20-, 40-, and 80-nm signal bandwidths. Raman amplifiers with equally spaced pumps and optimized central wavelength simplify system design while maintaining a gain ripple that is within $0.4 \mathrm{~dB}$ of the gain ripple of an amplifier with optimized pump wavelengths for the cases that was considered here.

\section{ACKNOWLEDGMENT}

The authors would like to thank V. Perlin and C.-J. Chen for many useful discussions.

\section{REFERENCES}

[1] H. Kidorf, "Recent advances in Raman amplification and applications," in Proc. Conf. Lasers and Electro-Optics 2001 (CLEO 2001), 2000, CTuJ3, p. 145.

[2] V. Perlin and H. Winful, "Optimal design of flat-gain wide-band fiber Raman amplifiers," J. Lightwave Technol., vol. 20, pp. 250-254, Feb. 2002.

[3] - "On distributed Raman amplification for ultrabroad-band long-haul WDM system," J. Lightwave Technol., vol. 20, pp. 409-416, Mar. 2002.

[4] S. Namiki and Y. Emori, "Ultrabroad-band Raman amplifiers pumped and gain-equalized by wavelength-division-multiplexed high-power laser diodes," IEEE J. Select. Topics Quantum Electron., vol. 7, pp. 3-16, Jan./Feb. 2001.

[5] K. Imai, I. Morita, H. Sakata, and N. Edagawa, "A study on gain modulation dynamics of $80 \mathrm{~nm}$-band high-power Raman amplifier," in Proc. Optical Fiber Communications Conf. (OFC 2003), 2003, TuC3, pp. $158-159$.

[6] H. B. Keller, Numerical Methods for Two-Point Boundary-Value Problems. Waltham, MA: Ginn and Blaisdell, 1968.

[7] H. Kidorf, K. Rottwitt, M. Nissov, M. Ma, and E. Rabarijanona, "Pump interactions in a 100-nm bandwidth Raman amplifier," IEEE Photon. Technol. Lett., vol. 11, pp. 530-532, May 1999.

[8] M. Karásek and M. Menif, "Channel addition/removal response in Raman fiber amplifiers: Modeling and experimentation," J. Lightwave Technol., vol. 20, pp. 1680-1687, Sept. 2002.

[9] S. J. Chen and W. S. Wong, "Transient effects in saturated Raman amplifiers," Electron. Lett., vol. 37, no. 6, pp. 371-373, Mar. 2001.

[10] Z. Michalewicz, Genetic Algorithms + Data Structures = Evolution Programs. New York: Springer-Verlag, 1992.

[11] G. Gonthier, "Novel fiber devices for Raman amplifiers and EDFAs," in Proc. Optical Fiber Communications Conf. (OFC 2003), 2003, WB6, pp. 310-311.

[12] G. P. Agrawal, Fiber Optic Communication Systems, 2nd ed. New York: Wiley-Interscience, 1997.

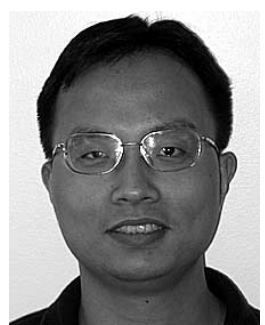

Jonathan Hu (S'00) received the B.S. degree in electrical engineering from Zhejiang University, Hangzhou, China, in 1997 and the M.S. degree from the University of Tulsa, Tulsa, OK, in 2001. He is currently working toward the Ph.D. degree at the University of Maryland Baltimore County (UMBC).

His research interests include modeling Raman amplifiers, erbium-doped fiber amplifiers, receivers, and microstructured optical fiber.

Mr. Hu is a Member of Tau Beta Pi, a Student Member of the Optical Society of America (OSA), and a Student Member of the IEEE Lasers \& Electro-Optics Society (LEOS).

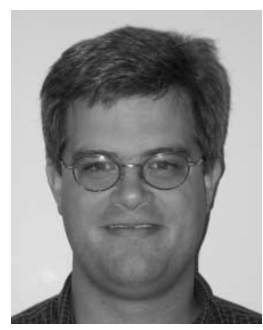

Brian S. Marks (M'03) received the B.S. degree in mathematics and physics from North Carolina State University, Raleigh, in 1995 and the Ph.D. degree in applied mathematics from Northwestern University, Evanston, IL, in 2000.

Since that time, he has been a Research Faculty Member of the Department of Computer Science and Electrical Engineering at the University of Maryland Baltimore County (UMBC). His research interests focus on the theoretical and computational study of light propagation in optical fibers.

Dr. Marks is a Member of the Optical Society of America (OSA) and the Society for Industrial and Applied Mathematics.

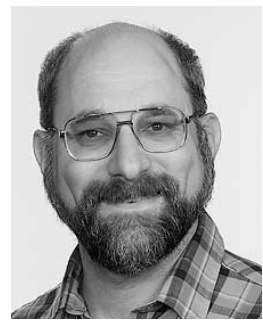

Curtis R. Menyuk (F'98) was born on March 26, 1954. He received the B.S. and M.S. degrees from the Massachusetts Institute of Technology (MIT), Cambridge, in 1976 and the $\mathrm{Ph} . \mathrm{D}$. degree from the University of California at Los Angeles (UCLA) in 1981.

$\mathrm{He}$ has worked as a Research Associate at the University of Maryland, College Park, and at Science Applications International Corporation, McLean, VA. In 1986, he became an Associate Professor in the Department of Electrical Engineering at the University of Maryland Baltimore County (UMBC), and he was the Founding Member of this department. In 1993, he was promoted to Professor. He was on partial leave from UMBC from fall 1996 until fall 2002. From 1996 to 2001, he worked part-time for the Department of Defense, codirecting the Optical Networking program at the DoD Laboratory for Telecommunications Sciences, Adelphi, MD, from 1999 to 2001. From 2001 to 2002, he was Chief Scientist at PhotonEx Corporation. For the last 15 years, his primary research area has been theoretical and computational studies of fiber optic communications. He has authored or coauthored more than 180 archival journal publications as well as numerous other publications and presentations. He has also edited two books. For the last 17 years, his primary research area has been theoretical and computational studies of fiber-optic communications. The equations and algorithms that he and his research group at UMBC have developed to model optical fiber transmission systems are used extensively in the telecommunications industry.

Dr. Menyuk is a Fellow of the Optical Society of America (OSA). He is a Member of the Society for Industrial and Applied Mathematics and the American Physical Society and a former UMBC Presidential Research Professor. 